\title{
The Use of Instrumentation in Grammar Engineering
}

\author{
Norbert Bröker \\ Eschenweg 3, 69231 Rauenberg
}

\begin{abstract}
This paper explores the usefulness of a technicue from software enginecring; code instrumentation, for the development of large-scalc natural language grammars. Information about the usage of grammar rules in test and corpus sentences is used to improve grammar and testsuite, as well as adapting a grammar to a specific genre. Results show that less than lialf of a large-coverage grammar for German is actually tested by two large testsuites, and that $10-30 \%$ of testing time is redundant. This methodology applied can be seen as a re-11se of grammar writing knowledge for testsuite compilation. Tho construction of genre-specific grammars results in performance gains of a factor of four.
\end{abstract}

\section{Introduction}

The field of Computational Linguisties (CL) has both moved towards applications and towards large data sets. These developments call for a rigorous methodology for creating so-called lingware: linguistic data such as lexica, grammars, trce-banlss, as woll as software processing it. Exporience from Software Engineering has shown that the earlier deficiencies are detected, the less costly their correction is. Rather than being a post-dovelopment effort, quality evaluation must be an integral part of development to make the construction of lingware more efficient (c.g., cf. (EAGLES, 1996) for a gencral cvaluation framework and (Ciravegna et al., 1998) for the application of a particular software design methodology to linguistic engineering). This paper presents the adaptation of a particular Software Engincering (SE) method, instrumentation, to Grammar Engineering (GE). Instrumentation allows to determine which test item exercises a certain piece of (software or grammar) code.

The paper first describes the use of instrumentation in SE, then discusses possible realizations in unification grammars, and finally presents two classes of applications.

\section{Software Instrumentation}

Systematic software testing requires a match between the test subject (module or complete system) and a test suite (collection of test items, i.e., sample input). This match is usually computed as the percentage of code items exerciscd by the test suite.

Depending on the definition of a code item, various measures are employed, for example (cf. (Hetzel, 1988) and (EAGLES, 1996, Appendix B) for overviews):

statement coverage percentage of single statemonts exercised

branch coverage percentage of ares exercised in control flow graph; subsumes statement coverage

path coverage percentage of paths exercised from start to end in control flow graph; subsumes branch coverage; impractical due to large (often infinite) number of paths

condition coverage percentage of (simple or aggregate) conditions evaluated to both true and false (on different test itcms)

Testsuites are constructed to maximize the targeted measure. A test run yields information about the code items not excrcised, allowing the improvement of the testsuite.

The measures are automatically obtained by instrumentation: The test subject is extended by code which records the code items exercised during processing. After processing the testsuite, the records are used to compute the measures.

\section{Grammar Instrumentation}

Measures from SE camnot simply be transferred to unification grammars, because the structure of (imperative) programs is different from (declarative) grammars. Nevertheless, the structure of a grammar (formalism) allows to define measures very similar to those employed in SE.

constraint coverage is the quotient

$$
T_{\text {con }}=\frac{\# \text { constraints exercised }}{\# \text { constraint in grammar }}
$$


where a constraint may be either a phrasestructure or an equational constraint, depending on the formalism.

disjunction coverage is the guotiont

$$
T_{\text {dis }}=\frac{\# \text { disjunctions covered }}{\# \text { disjunctions in grammatr }}
$$

where a disjunction is considered covered when all its alternative disjuncts have been separately exercised. It encompasses constraint coverage. Optional constituents and equations have to be troated as a disjunction of the constraint and an empty constraint (cf. lig.2 for an oxample).

interaction coverage is the quoticnt

$$
T_{\text {int }}=\frac{\# \text { disjunct combinations exoreised }}{\# \text { legal disjunct combinations }}
$$

where a disjunct combination is a complete sot of choices in the disjunctions which yields a wellformed grammatical structure.

As with path coverage, the set of legal disjunct combination typically is infinite clue to recursion. A solution from Sls is to restrict the use of recursive rules to a fixed number of cases, for (xample not using the rule at all, and using it only once.

Tho goal of instromentation is to olotain informattion about which test cases exereise whicle granmant constraints. Ono way to record this infommation is to extend the parsing algorithm. Another way is to 1 so the grammat fomalism it self to ielentify the disjuncts. Depending on the experessivity of the formalism used, the following pessibilities exist:

atomic features Assuming a unique numbering of disjuncts, an anmotation of the form DISJUNCT-nn $=+$ can bo used for matking. 'To determine whether a certain disjunct, wats used in constructing a solution, one only needs to check whether the associated faiture occurs (at some level of embodeling) in the solntion.

set-valued features If set-valued foutures are availalsle, one can use a set-ralued feature DISJUNCTS to collect atomice symbols representing one disjunct each: DISJUNCT-nn $\in$ DISJUNCTS, which might case the collection of exercised clisjuncts.

multiset of symbols The recover the number of times a clisjunct is used, one need is to leave the mification paradign, because it is very diflicult, to comnt with minfication grammats. Wo have used a special foature of om grammate developenent environnent; Following the LIF spirit of clifferent projections, it provicles a projection for

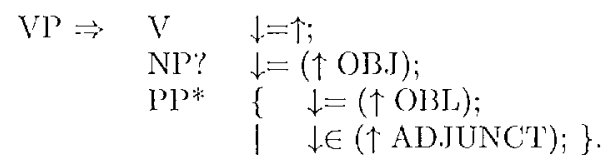

Jigure 1: Sample Rule

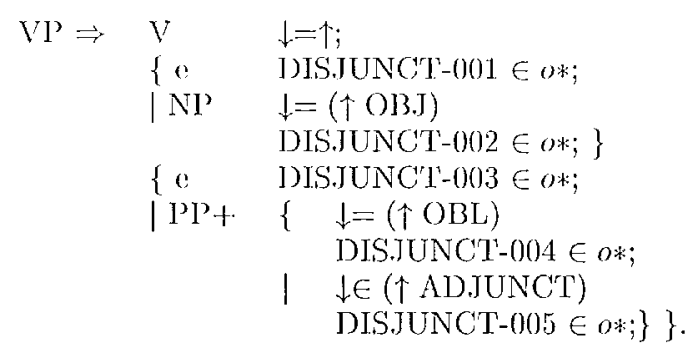

Figure 2: Instrumentied rule

symbolic manks, which is formally equivalont to a nunltised of symbols associated with the complete solution (structural embedding plays no role; seo (liank ot al., 1998) for applications). In this way, we can collect from the root node of oach solution the set of all disjuncts exereised, together with a lisage count.

Consider the Llig grammar rule in Fig.1.' Constraint coverage would require test items such that overy eategory in the VP is excereised; a secuence of V NP PP would suflice for this measure. J)isjunction coverage also recpuires to take the empty disjuncts into account: NP and PP are optional, so that four items are needed to achicve full disjunction coverage on the phatse structure prat of the rule. I) lae to the disjunetion in the PP amotation, two more test items are required to achieve full disjunction coverage on the complete rule. Fig.2 shows the rule from Fig.1 with instrumentation.

\section{Grammar and Testsuite Improvement}

Traditionally, a testsuite is used to improve (or maintain) a grammats's quality (in tems of coverage and overgeneration). Using instrumentation, one may extend this usage by looking for sources of

\footnotetext{
Although the sample rule are in the format of J FG, nothing of the nethodology relies on the choice of linguistic or conputational paradign. The notation: ? $/ * /+$ represent op tionality/itoration including/excluding zero occurrences on alegories. e represents the cmpty string. Anmotations to a category specify exuality $\Leftrightarrow$ or set membership (G) of feature values, or non-existence of features $(\neg)$; they are terminated by a semicolon (;). Disjunctions are given in braces $(\{\ldots \mid \ldots\}) . \uparrow(\downarrow)$ are notavariables representing the foafure structure corresponding to the mother (danghter) of the rule. $\theta *$ (lor optinality) represents the sentence's multi-set valued symbolic projection. Comments are onelosed in (juotation marks (".."). Cf. (Kaplan and Bresnan, 1082) for an introchetion to $\mathrm{B} \mathrm{l}$ G notation.
} 
overgencration (cf. Sec.4.3), and may also improve the quality of the testsuite, in terms of coverage (cf. Sec.4.1) and economy (cf. Sec.4.2).

Complementing other work on testsuite construction (cf. Sec.4.4), I will assume that a grammar is already available, and that a testsuite has to bo constructed or extended. While one may argue that grammar and testsuite should be developed in parallel, such that the coding of a new grammar disjunct is accompanied by the addition of suitable test cases, and vice versa, this is seldom the case. Apart from the existence of grammars which lack a testsuite, there is the more principled obstacle of the evolution of the grammar, leading to states where previously necessary rules silently loose their usefulness, because their function is taken over by some other rules, structured differently. This is detectable by instrumentation, as discussed in Sec.4.1.

On the other hand, once there is a testsuite, it has to be used economically, avoiding redundant tests. Sec.4.2 shows that there are different levels of redundancy in a testsuite, dependent on the specific grammar used. Reduction of this redundancy can speed up the test activity, and give a clearer picture of the grammar's performance.

\subsection{Testsuite Completeness}

If the disjunction coverage of a testsuite is 1 for some grammar, the testsuite is complete w.r.t. this grammar. Such a testsuite can reliably be used to monitor changes in the grammar: Any reduction in the grammar's coverage will show up in the failure of some test case (for negative test cases, cf. Sec.4.3).

If the testsuite is not complete, instrumentation can identify disjuncts which are not exercised. These might be cither (i) appropliate, but untested, disjuncts calling for the addition of a test case, or (ii) inappropriate disjuncts, for which a grammatical test casc exercising them cannot be constructed.

Checking completeness of our local testsuite of 1787 items, we found that only 1456 out of 3730 grammar disjuncts in our German grammar were tested, yielding $T_{\text {dis }}=0.39$ (the TSNLP testsuite containing 1093 items tests only 1081 disjuncts, yielding $\left.T_{\text {lis }}=0.28\right){ }^{2}$ Fig.3 shows an cxample of a gap in our testsuite (there are no examples of circumpositions), while Fig.4 shows an inapproppriate disjunct thus discovered (the category ADVadj has been eliminated in the lexicon, but not in all rules). Another crror class is illustrated by Fig.5, which shows a disjunct that can never be used due to an LFG colierence violation; the grammar is inconsistent here. ${ }^{3}$

\footnotetext{
${ }^{2}$ There are, of course, unparsed but grammatical test cases in both testsuites, which have not been taken into account in these figures. This explains the difference to the overall number of 1582 items in the German TSNLP testsuite.

${ }^{3}$ Test cases using a free dative pronoun may be in the test-
}

$$
\begin{array}{lll}
\text { PPstd } \Rightarrow & \text { Pprac } & \downarrow=\uparrow ; \\
& \text { NPstd } & \downarrow=(\uparrow \text { OBJ }) ; \\
& \text { \{e } & \text { DISJUNCT-011 } \in o * ; \\
& \mid \text { Pcircum } & \downarrow=\uparrow ; \\
& & \text { DISJUNCT-012 } \in o^{*} \\
& & \text { "unused disjunct"; }\}
\end{array}
$$

Figure 3: Appropriate untested disjunct

$$
\begin{aligned}
& \mathrm{ADVP} \Rightarrow\{\{\mathrm{e} \quad \text { DISJUNCT-021 } \in \text { o* } \\
& \text { | ADVadj } \downarrow=\uparrow \\
& \text { DISJUNCT-022 } \in \text { o* } \\
& \text { "unused disjunct"; \} } \\
& \text { ADVstd } \downarrow=\uparrow \\
& \text { DISJUNCT-023 } \in \circ * \\
& \mid \ldots\} \text {. } \\
& \text { "unused disjunct"; \} }
\end{aligned}
$$

Figure 4: Inappropriate disjunct

\subsection{Testsuite Economy}

Besides being complete, a testsuite must be conomical, i.e., contain as few items as possible. Instrumentation can identify redundant test cases, wherc redundancy can be defined in three ways:

similarity There is a set of other test cases which jointly exercise all disjunct which the test case under consideration exercises.

equivalence There is a single test case which cxercises exactly the same combination(s) of disjuncts.

strict equivalence There is a single test case which is equivalent to and, additionally, exercises the disjuncts exactly as often as, the test case under consideration.

Fig.6 shows equivalent test cases found in our testsuite: Example 1 illustrates the distinction between equivalence and strict equivalence; the test cases contain different numbers of attributive adjectives. Example 2 shows that our grammar does not make any distinction between adverbial usage and secondary (subject or object) predication.

The reduction we achieved in size and processing time is shown in Table 1, which contains measurements for a test run containing only the parseable test cases, one without, equivalent test cases (for evcry set of equivalent test cases, one was arbitrarily selected), and one without similar test cases. The last was constructed using a simple heuristic: Starting with the sentence exercising the most disjuncts, working towards sentences relying on fewcr disjuncts, a sentence was selected only if it exercised a disjunct which no previously selected sentence exercised. Assuming that a disjunct working correctly

suite, but receive no analysis since the grammatical function FREEDAT is not defined as such in the configuration section. 


$$
\begin{aligned}
& \text { VPargs } \Rightarrow\left\{\begin{array}{l}
\text {.. } \\
\text { PRONstel } \downarrow=(\uparrow \text { FREEDA' })
\end{array}\right. \\
& (\downarrow \mathrm{CASS})=\text { dat } \\
& (\downarrow \text { PRON-TYPJ })=\text { pers } \\
& \neg(\uparrow \text { ()B.J2) } \\
& \text { DIS.JUNCT'-(0)4 } \in o * \\
& \mid \ldots\} \text {. } \\
& \text { "unused disjunct"; }
\end{aligned}
$$

Figure 5: Inconsistent disjunct

1 ein guter alter Wein ein guter alter trockener Wein 'a good old (dry) wine'

2 Er ißt das Schnitzel roh.

Fr ißt das Schnitzol nackt.

Er ißt das Schnitzol schncll.

'He cats the schnitzel naticd/raw/quichly.'

Figure 6: Sets of equivalent test cases

once will work correctly more than once, we did not consider strict equivalence.

We envisatge the following nes of this redundancy detection: 'I'here clearly aro linguistic: reasons to distinguish all lest cases in example 2, so they (anmot simply be deleted from the testsuite. Rather, their equivalence indicates that the grammare is not yet perfect (or never will be, if it remains purely syntactic). Such equivalences could bo interpreted as a reminder which linguistic distinctions need to be incorporated into the grammmer. Thes, this level of redumdancy may drivo your grammal devolopment, agenda. 'Tho level of equivatenes an be tation as a limited interaction test: Thuse test anes nepresent one complete selection of gramman disjuncts, and (given the grammar) there is nothing wo can gain by checking a test caso if an equivalent one was tested. Thus, this lovel of redundancy may be used for ensuring the quality of grammar changes prior

\begin{tabular}{|c|c|c|c|c|}
\hline & $\begin{array}{l}\text { test } \\
\text { calses }\end{array}$ & $\begin{array}{c}\text { relative } \\
\text { size }\end{array}$ & $\begin{array}{c}\text { runtine } \\
\text { (sec) }\end{array}$ & $\begin{array}{l}\text { relative } \\
\text { runtime }\end{array}$ \\
\hline \multicolumn{5}{|c|}{ TSNI 3 testsuite } \\
\hline parscable & 1093 & $100 \%$ & 1537 & $100 \%$ \\
\hline no equivalents & 783 & $71 \%$ & 665.3 & $43 \%$ \\
\hline no similar cases & 214 & $19 \%$ & 128.5 & \\
\hline \multicolumn{5}{|c|}{ local testsuite } \\
\hline parscable & 1787 & $100 \%$ & 1213 & 1.00 \\
\hline no equivalents & 1600 & $89 \%$ & 899.5 & 74 \\
\hline no similar cascs & 331 & $18 \%$ & 175.0 & 14 \\
\hline
\end{tabular}
to their incolporation into the production version of the grammas. 'The level of similarity contatins much less test cases, and cloes not test any (systematic) interaction between disjuncts. Thus, it may be used during development as a quick lule-of-thumb procedure detecting serions orrors only.

Table 1: Reduction of 'Lestsuites

$\begin{array}{ll}\text { Der Tost fällt leicht. } & \text { Dieselbe schlafen. } \\ \text { Die schlafen. } & \text { Das schlafen. } \\ & \text { Eines schlafen. } \\ \text { Man schlafon. } & \text { Jede schlafen. } \\ \text { Dieser schlafen. } & \text { Dieses schlafen. } \\ \text { Ich schlafen. } & \text { Eine schlafon. } \\ \text { Jer schlafen. } & \text { Meins schlafen. } \\ \text { Jerler schlafen. } & \text { Dajonige schlafen. } \\ \text { Derjonige schlafen. } & \text { Jedes schlafon. } \\ \text { Jener schlafen. } & \text { Diejenige schlafen. } \\ \text { Keincr schlafen. } & \text { Jenes schlafen. } \\ \text { Derselbe schlafen. } & \text { Keines schlafen. } \\ \text { Jis schlafen. } & \text { Dasselbe schlafen. } \\ \text { Irgendjemand schlafen. } & \end{array}$

ligure 7 : Sentences relying on suspicious disjunct

\subsection{Sources of Overgeneration}

To control overgencration, appropriately marked ungrammatical sentences are important in every testsuite. Instrunentation as proposed here only looks at successful parses, but (an still be applied in this context: If an ungrammatical test case receives an analysis, instrumentation informs us about the disjuncts used in the incorrect analysis. One of these disjuncts must be incorrect, or the sentence would not have received a solution. We exploit, this information by accumulation across the entine test suite, looking for disjuncts that appear in unusually high proportion in parseable ungrammatical test cuses.

In this mannor, six grammar disjuncts are singled out by the parseable ungrammatical test cases in the 'TSNIP testsuite. The most prominent disjunct appenes in 26 sentences (listed in lig. 7 ), of which the top left group is inded grammatical and the rest fall into two chases: A partial VP with object $\mathrm{NP}$, interpreted as an imporative sentence (bottom left), and a woird interaction with the tokenizer incorrectly handling capitalization (right group).

Far from boing conclusive, the similarity of these sentences derived from a suspicious grammar disjunct, and the clear relation of the sentences to only two exactly specifiable grammar crors make it plausilsle that this approach is very promising in cletecting the sourcess of overgencration.

\subsection{Other Approaches to Testsuite Construction}

The delicacy of testsuite construction is acknowlodged in (EAGLES, 1996, p.37). Although there are a number of efforts to construct reusable testsuites, none has to my linowledge explored how existing grammars can be exploited.

Starting with (Flickinger of al., 1987), testsuites have becen drawn up from a linguistic viewpoint, informed by [the] study of linguistics and [roflecting] the grammatical issues that linguists have concerned themselwes with (Flickinger et al., 1987, p.4). Al- 
though the question is not explicitly addressed in (Balkan, 1994), all the testsuites reviewed there also seem to follow the same methodology. The TSNLP project (Lehmann and Oepen, 1996) and its successor DiET (Notter ot al., 1998), which built large multilingual testsuites, likewise fall into this category.

The use of corpora (with various levels of annotation) has becn studied, but the recommendations are that much manual work is recpuired to turn corpus cxamples into test cases (e.g., (Balkan and Fouvry, 1995)). The reason given is that corpus sentences neither contain linguistic phonomena in isolation, nor do they contain systematic variation. Corpora thus are used only as an inspiration.

(Oepen and Flickinger, 1998) stress the interdependence between application and testsuite, but don't comment on the relation between grammar and testsuite.

\section{Genre Adaptation}

A different application of instrumentation is the tailoring of a gencral grammar to specific genres. Allpurpose grammars are plagued by lexical and structural ambiguity that leads to overly long runtimes. If this ambiguity could be limited, parsing efficiency would improve. Instrumenting a gencral grammar allows to automatically derive specialized subgrammars based on sample corpora. This setup has sevcral advantages: The larger the overlap between genres, the larger the portion of grammar development work that can be recycled. The all-purpose grammar is linguistically more interesting, because it requires an integrated concept, as opposed to several separ rate genre-specific grammars.

I will discuss two ways of improving the efficiency of parsing a sublanguage, given an all-purpose unification grammar. The first consists in deleting unused disjuncts, while the second uses a staged parsing process. The experiments are only sketched, to indicate the applicability of the instrumentation technique, and not to directly compete with other proposals on grammar specialization. For example, the work reported in (Rayner and Samuelsson, 1994; Samuclsson, 1994) differs from the one presented below in several aspects: They induce a grammar from a treebank, while I propose to annotate the grammar based on all solutions it produces. No criteria for tree docomposition and category specialization are needed here, and the standard parsing algorithm can be used. On the other hand, the efficiency gains are not as big as those reported by (Rayner and Samuelsson, 1994).

\subsection{Restricting the Grammar}

Given a large sample of a genre, instrumentation allows you to determine the likely constructions of that genre. Eliminating unused disjuncts allows faster

\begin{tabular}{llc} 
Descriptor & Content & Coverage \\
\hline HC-DE & Copier/Printer User Manual & $89 \%$ \\
WHB & Car Maintenance Instructions & $76 \%$ \\
NEWS & News (5-30 words per sentence) & $42 \%$ \\
NEWS-SC & Verb-final subclauses from News & $75 \%$
\end{tabular}

Table 2: Corpora used for adaptation

parsing due to a smaller grammar. An experiment was conducted with several corpora as detailed in Table 2. There was some eflort to cover the corpus HC-DE, but no grammar development based on the other corpora. The NEWS-SC corpus is part the corpus of verb-final sentences used by (Beil ct al., 1999).

A training set of 1000 sentences from each corpus was parsed with an instrumented base grammar. From the parsing results, the exercised grammar disjuncts were extracted and used to construct a corpus-spocific reduced grammar. The reduced grammars were then used to parse a test set of another 1000 sentences firom each corpus. Table 3 shows the performance improvement on the corpora: It gives the size of the grammars in terms of the number of rules (with regular expression right-hand sides and feature annotation), the number of ares (corresponding to unary or binary rules with disjunctive feature annotation), and the number of disjuncts (unary or binary rules with unicue feature annotation). The number of mismatches counts the sentences for which the solution(s) obtained differed from those obtained with the base grammar, while the number of additions counts the sentences which did not receive a parse with the base grammar due to resource limitations (runtine or memory), but received one with the reduced grammar. The other columns give timings to process the total corpus, and the longest and average processing time per sentence; time is in soconds. The last column gives the average number of solutions per sentence.

Due to the sampling of a genre, the grammars obtained can only be approximate. To determine the relation of the sample size to the cuality of the grammar obtained, the coverage of random fragment grammars was measured in the following way: Randomly select a number: of sentences from the total corpus, construct (in the same way as described above for the reduced grammar) a fragment grammar, and determine its coverage on the test set from the respective corpus. The graphs in Fig.8 show how the coverage and runtime relate to the number of sentences on which the fragment grammars are based. 'The leftmost data point ( $x$ value 0 ) describes the performance of the reduced grammar on the training set, while the rightmost clata point clescribes its performance on the test set. The data points in between represent fragmont grammars based on as 


\begin{tabular}{|c|c|c|c|c|c|c|c|c|c|}
\hline & 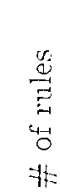 & 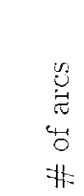 & 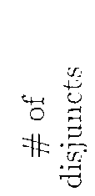 & 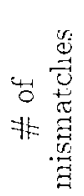 & 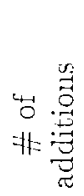 & 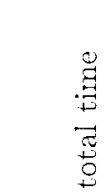 & 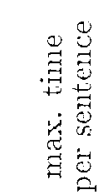 & 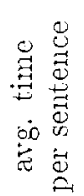 & 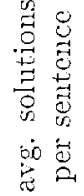 \\
\hline Corpus HC-DE & & & & & & & & & \\
\hline base grammar & 185 & 3669 & 11564 & $11 / a$ & $11 / a$ & 7692.4 & $>300$ & 7.1 & 10.1 \\
\hline rerluced grammar (938) & 112 & 960 & 3739 & 0 & 1 & 2089.4 & 162.7 & 1.9 & 17.6 \\
\hline Corpus WIII3 & & & & & & & & & \\
\hline base grammar & 195 & 3728 & 1.1600 & $11 / a$ & & 1428.9 & $>300.3$ & 1.5 & \\
\hline reluced grammar (559) & & 534 & 3072 & 1 & & 1414.2 & 11.3 & 0.4 & \\
\hline
\end{tabular}

Jable 3: Jerformance of reduced grammats
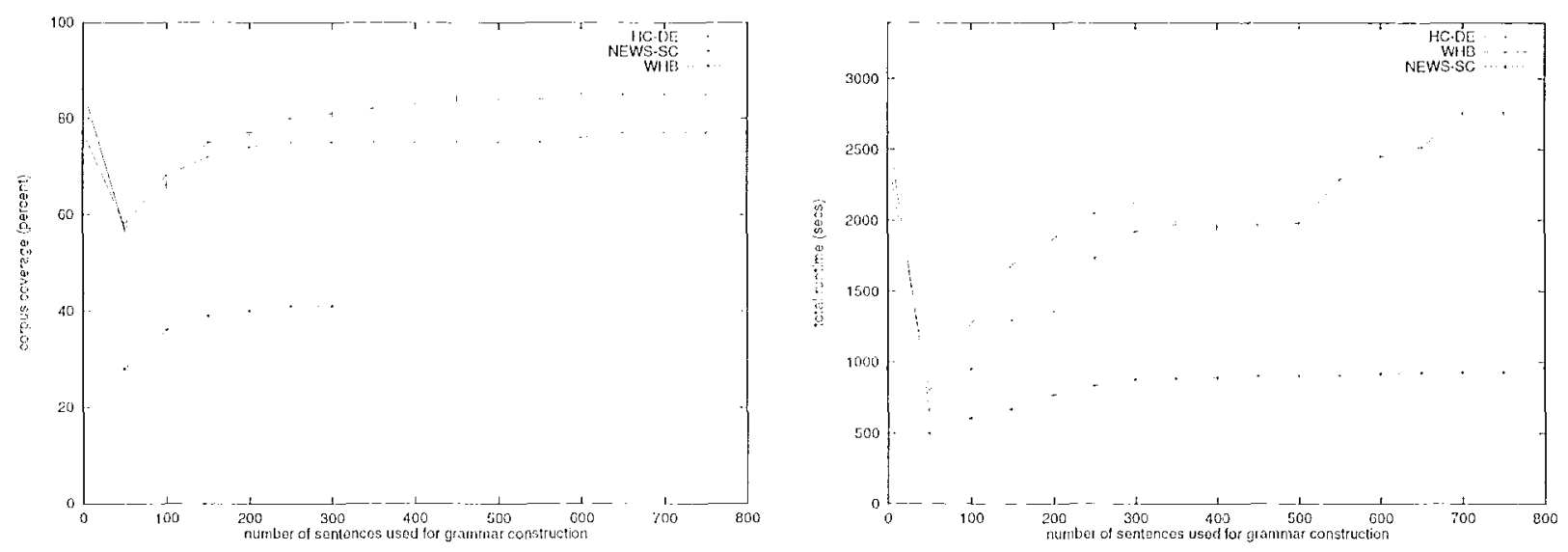

Figure 8: Performance of fiagment grammars

naluy sentences as given by the $\mathrm{x}$ axis value.

The resultis enorted here represent tho minimal performance gatin due to the fact that the construetion of reduced and fiagnent grammans are not based on the conect solutions for the tanining sentonces, but rather on all solutions produced by the base grammar. The construction of a large-scale tirechank with manually verified solutions is under way but lats not yet progressed far enough to serve as inpot for this experiment. Even with this systenatic, but curable error, the reduction reduces overall processing by a factor of four. 'The number of solutions is constant becauso only tunused disjuncts are eliminated; this will change if the treebank solntions are used to construct the roluced grammar.

\subsection{Staged Parsing}

Even oliminating only unlikely disjuncts necessarily reduces the corerage of the grammat: A secuence of parsing stages allows one to profit from a sinall and fast granmar as woll as from a large and slow one. Staged parsing applies different grammars one after the other to the input, until one yiclds a solution, which terminates the process. In our case, a grammar of stage $n+1$. includes the grammar of stige $n$, but this need not be the catse in general.
Tor reduce the variability for an experiment, I assume three stages: The first includes ficepuently used disjuncts, the second infrequent disjuncts, and the thined mused disjunets. 'J'his ensures the full coverage of the base gramman, but allows to focus on fre(puent constructions in the first parsing stage. The procedure is similar as before: from the solutions of a traningerset, a staged grammar is constructed. Currently, experinents are performed to determino a useful dofinition of 'frequently used'. Independent from the actual performance gains finally obtained, the application of instrunentation allows a systematic exploration of the possible configurations.

\subsection{Other approaches to grammar adaptation}

(Rayner and Samuelsson, 1994; Raynor and Carter, 1996; Samuelsson, 1994) present a grammar specialization technicue for mification grammars. From a trecbank of the sublanguage, they induce a specialized grammar using fewer macro vules which correspond to the application of several original rules. They report an average specd-up of 55 for only the parsing phase (taking lexical lookup) into account, the speed-1ep factor was only 6-10). Due to tho derivation of the grammar from a corpus sample, 
they observed a decrease in recall of $7.3 \%$ and an increase of precision of $1.6 \%$. The differences to the approach described here are clear: Starting from the grammar, rather than from a treebank, we annotate the rules, rather than inducing them from scratch. We do not need criteria for tree decomposition and category specialization, and we can use the standard parsing algorithm. On the other hand, the efficiency gains are not as big as those reported by (Rayner and Carter, 1996) (but note that we cannot measure parsing times alone, so we need to compare to their speed-up factor of 10). And we did not (yet) start from a treebank, but from the raw set of solutions.

\section{Conclusion}

I have presented the adajtation of code instrumentation to Grammar Enginecring, discussing measures and implementations, and sketching several applications together with preliminary results.

The main application is to improve grammar and testsuite by exploring the relation between both of them. Viewed this way, testsuite writing can benefit from grammar development bocause both describe the syntactic constructions of a natural language. Testsuites systematically list these constructions, while grammars give generative procedures to construct them. Since there are currently many more grammars than testsuites, we may re-use the work that has gone into the grammars for the improvement of testsuites.

Other applications of instrumentation are possible; genre adaptation was discussed in some depth. On a more general level, onc may ask whether other methods from SE may fruitfully apply to GE as well, possibly in modified form. For example, the static analysis of programs, e.g., detection of umeachable code, could also be applied for grammar development to detect unusable rules.

\section{References}

L. Balkan and F. Fouvry. 1995. Corpus-based test suite generation. TSNLP-WP 2.2, University of Essex.

I. et al. Ballan. 1994. Test Suite Design Annotation Scheme. TSNLP-WP2.2, University of Essex.

F. Beil, G. Carroll, D. Prescher, S. Riezler, and M. Rooth. 1999. Inside-outside estimation of a lexicalized PCFG for german. In Proc. 37th Annual Meeting of the ACL. Maryland.

F. Ciravegna, A. Lavelli, D. Petrelli, and F. Pianesi. 1998. Developing language reesources and applications with GEPPETTO. In Proc. 1st Int'l Conf. on Language Resources and Evaluation, pages 619-625. Granada/Spain, 28-30 May 1998.

EAGLES. 1996. Evaluation of Natural Language Processing Systems. Final Report EAG-EWGPR.2.
D. Flickinger, J. Nerbonne, I. Sag, and T. Wasow. 1987. Toward Evaluation of NLP Systems. Hewlett-Packard Laboratories, Palo Alto/CA.

A. Frank, T.H. King, J. Kuhn, and J. Maxwell. 1998. Optimality theory style constraint ranking in large-scale LFG grammar. In Proc. of the LFG98 Conference. Brisbane/AUS, Aug 1998, CSLI Online Publications.

W.C. Hetzel. 1988. The complete guide to software testing. QED Information Sciences, Inc. Wellesley/MA 02181.

R.M. Kaplan and J. Bresnan. 1982. Lexicalfunctional grammar: A formal system for grammatical representation. In J. Bresnan and R.M. Kaplan, cditors, The Mental Representation of Grammatical Relations, pages 173-281. Cambridge, MA: MIT Press.

S. Lehmann and S. Oepen. 1996. Tsnlp - test suites for natural language processing. In Proc. 16th Int'l Conf. on Computational Linguistics, pages 711-716. Copenhagen/DK.

K. Netter, S. Armstrong, T. Kiss, J. Klein, and S. Lehman. 1998. Dict - diagnostic and evaluation tools for nlp applications. In Proc. 1st Int'l Conf. on Languagc Resources and Evaluation, pages 573-579. Granada/Spain, 28-30 May 1998.

S. Oepen and D.P. Flickinger. 1998. Towards systematic grammar profiling:test suite techn. 10 years afte. Journal of Computer Speech and Langrage, 12:411-435.

M. Rayner and D. Carter. 1996. Fast parsing using pruning and grammar specialization. In Proc. 34th Annual Mecting of the ACL. Santa Cruz, USA.

M. Rayner and C. Samuelsson. 1994. Corpus-based grammar specialization for fast analysis. In M.S. Agnäs, H. Alshawi, I. Btrean, D. Carter, and K. Coder, editors, Spoken Language Translator: First-Year Report, pages 41-54. Report CRC-043, Cambridge/UK: SRI International.

C. Samuelsson. 1994. Grammar spezialization through cntropy thresholds. In Proc. 32nd Annual Meeting of the ACL. 